\title{
Analysis of Structural Variations of the Precipitate Based on Monitoring the Industrial Electrolysis of Copper Powders of Various Brands
}

\author{
E. E. Sokolovskaya ${ }^{a, b}, *$ M. L. Osipova ${ }^{a, b}$, I. B. Murashova ${ }^{b, * *}$, A. B. Darintseva ${ }^{b, * * *}$, \\ A. M. Savel'ev ${ }^{a}$, and F. F. Mukhamadeev ${ }^{b}, * * *$ \\ ${ }^{a}$ OAO Uralelektromed', ul. Lenina 1, Verkhnyya Pyshma, Sverdlovsk oblast, 624090 Russia \\ ${ }^{b}$ Ural Federal University, ul. Mira 19, Yekaterinburg, 620002 Russia \\ *e-mail: E.Sokolovskaya@elem.ru \\ **e-mail:el-chem@mail.ustu.ru,mirib@rambler.ru \\ ***e-mail:el-chem@mail.ustu.ru
}

\begin{abstract}
This study is associated with the solution to the blending problem of the charge for the output of ready articles. The main requirements are claimed to the specific surface and the packed density of the powders of basic brands PML0, PMS-1, and GG. The dynamics of the development of the dendrite depositions for powders of mentioned brands with the simultaneous registration of the cathodic overvoltage is investigated under industrial experimental conditions. The procedure is developed and the results of the continuous monitoring of the deposition growth with the direct immersion of a video camera into the electrolyte are presented. The reproducibility of the results is evaluated statistically. Based on the galvanostatic crystallization model of the dendrite deposition on the rod electrode, the dynamics of varying the density $(N)$ and radius $\left(r_{\mathrm{t}}\right)$ of growing tips on the growth front is calculated. It is established by variance analysis that structural parameters $N$ and $r_{\mathrm{t}}$ are individual for depositions of every brand, so that the preparation conditions of the deposition during the electrolysis should be strictly fulfilled as the charge forms.
\end{abstract}

Keywords: growth dynamics, copper powder, electrocrystallization, photographing the deposition, overvoltage recording, tips of growing dendrites, density of tips on the deposition growth front, various powder brands, variance analysis

DOI: $10.3103 / \mathrm{S} 1067821213060291$

\section{INTRODUCTION}

In connection with the good compressibility of dendritic particles, copper electrolyte powders are widely used in powder metallurgy. The structure of dendritic depositions, which is usually presented by the granulometric composition of the powder and its specific surface, is especially important for the formation of the charge when producing wares with specified properties. Irrespective their brand, the powders are produced during electrolysis in conditions of deep diffusion control, namely, at the identical current load [1], which substantially (by a factor of $K_{\mathrm{d}}$ times) exceeds the corresponding limiting current. One of the problems of the investigation is in the comparative statistic analysis of the crystallization dynamics of dendritic depositions for powders of various brands with the overwhelming diffusion control of the electrolysis stage. If particles of one size of various brands have their structural features, the electrolysis stage is determining in the formation of the required structure. Precisely for this reason, for example, the shrinkages of wares with the same granulometric composition of the charge depend on the powder brand for which the electrolysis was performed [2]. The statistic comparison of the growth dynamics of dendrites is based on the electrolysis model of the dendritic deposition on the rod electrode [3].

\section{PROCEDURE OF COMPARING THE STRUCTURE OF DEPOSITIONS FOR VARIOUS BRANDS OF POWDERS}

The experimental investigation into the growth dynamics of the dendritic copper deposition was performed in conditions of the industrial production of copper powders of brands PML0, PMS-1, and GG at OAO Uralelektromed' [4] (Fig. 1). During deposition growth, the cathodic overvoltage relative to a copper spiral immersed into the electrolyte was registered using an APPA multimeter with a step of $10 \mathrm{~s}$. Before deposition removal, the array of measured values was transferred into the computer memory and these data were processed using Excel. The formation dynamics of the structure of powders of various brands was acquired periodically (with a step of 15-20 min) lifting a 


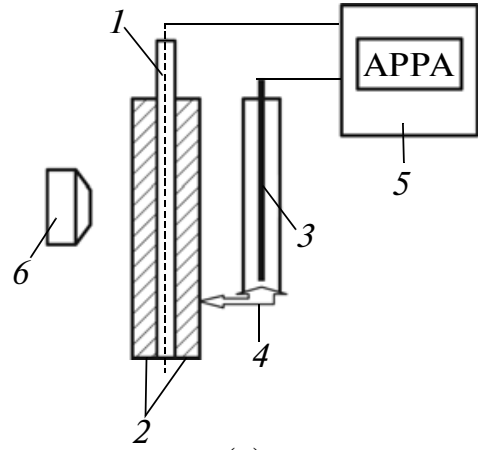

(a)

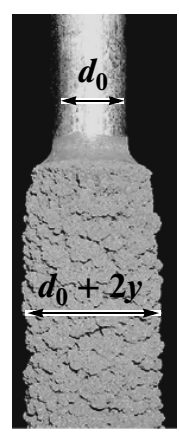

(b)
Fig. 1. (a) Measurement layout and (b) appearance of the cathode with the deposition when studying the dynamics of the deposition growth. (1) Cathode rod, (2) dendritic deposition of the cathode, (3) copper reference electrode, (4) glass key; (5) APPA multimeter, and (6) photocamera.

cathodic bar over the bath and photographing the cathode with the precipitate (Fig. 1b). The bar of one of neighboring baths, which was not previously used for photographing, was selected for each measurement.

The acquired information was used to evaluate the structural variations of the dendritic deposition.

\section{MODEL NOTIONS}

The length of developing dendrites $y$ (or the thickness of the layer of a loose deposition) on a cylindrical electrode with diameter $d_{0}$ with specified depletion coefficient $K_{\mathrm{d}}$ and the stationary limiting current density $i_{\text {lim }}$ varies [3] in time $t$ according to the expression

$$
d_{0} y+y^{2}=\frac{V}{z F} \frac{K_{\mathrm{d}} d_{0} i_{\mathrm{lim}}}{2 \pi N r_{\mathrm{t}}^{2}} t+B
$$

where $V$ is the molar volume of metal; $B=d_{0} y_{0}+y_{0}^{2}$ is the constant, which characterizes the magnitude of the initial nonuniformity of the electrode surface $y_{0}$; $z$ is the charge of copper ions; and $F$ is the Faraday constant. Quantity $N r_{\mathrm{t}}^{2}$ is the structural characteristic of the dendritic deposition, in which $N$ is the density of developing tips at the deposition growth front and $r_{\mathrm{t}}$ is the radius of these tips. It was established experimentally [5] that, during the electrocrystallization of the dendritic copper deposition, the characteristic $N r_{\mathrm{t}}^{2}$ varies slightly, which does not exclude time dependences $N(t)$ and $r_{\mathrm{t}}(t)$. Other parameters in the right side of Eq. (1) are time-independent. The hydrogen liberation parallel with the loose copper deposition, which increases the convective limiting current density of copper and, consequently, the current yield, forces us to introduce the current yield $\left(B_{\mathrm{c}}\right)$ as the multiplier into the first summand of the right side of Eq. (1). According to the manufacturing practice of electrolytic copper powders $\left(B_{\mathrm{c}}=90-95 \%\right)$, Eq. (1) takes the form

$$
d_{0} y+y^{2}=\frac{V}{z F} \frac{K_{\mathrm{d}} d_{0} i_{\lim } \times 0.95}{2 \pi N r_{\mathrm{t}}^{2}} t+B .
$$

There is a crystallization model of the dendritic deposition which involves parallel cathodic hydrogen liberation; however, it does not take into account the enhancement of the convective diffusion of metal ions to the cathode caused by this reaction. The connection between the hydrogen liberation rate and the effective thickness of the diffusion layer is taken into account in [6], but the empiric relationship between them should be refined.

The derivative with respect to time of the left part of Eq. (2) is described by the relationship

$$
\frac{d}{d t}\left(d_{0} y+y^{2}-B\right)=\frac{1}{N r_{\mathrm{t}}^{2}} \frac{V}{z F} \frac{K_{\mathrm{d}} d_{0} i_{\lim } \times 0.95}{2 \pi} .
$$

If the structural parameter of the dendritic precipitate $N r_{\mathrm{t}}^{2}$ is actually invariable during electrolysis, the derivative remains constant while Eq. (2) is illustrated by the straight line in coordinates $\left(d_{0} y+y^{2}-B\right), t$. Thus, relationship (3) allows us to determine the character of varying the structural characteristic of the dendritic deposition by analyzing the experimental $t$ dependences of $\left(d_{0} y+y^{2}-B\right)$. The measurement of the cathodic overvoltage $(\eta)$ during the electrolysis makes it also possible to find the separate characteristics $N(t)$ and $r_{\mathrm{t}}(t)$ with a mixed process control at dendritic tips.

\section{RESULTS OF MEASUREMENTS}

The growth dynamics of copper dendritic deposition of three brands (Fig. 2) is found based on processing the measurements of $\eta(t)$, photo-and-video materials. The scale of the increment of the deposition layer depends slightly on the powder brand. The maximal electrode diameter with the precipitate of the PML0 brand is $28 \mathrm{~mm}$, that of the PMS-1 brand is $29 \mathrm{~mm}$, and that of the GG brand is of $25 \mathrm{~mm}$. In this case, the thickening rate of the dendritic layer is radically determined by the powder brand. For example, the deposition development period for the PML0 brand is shorter by a factor of 2 compared with other two materials. The crystallization chronopotentiograms for the depositions of discussed brands (by the initial overvoltage after shaking off the deposition from the cathode, by the final overvoltage of growing the deposition on the cathode, and by the potential oscillation amplitude in the crystallization period of dendrites) differ substantially. The highest overvoltage accompanies the formation of the precipitate of the PML0 brand (from 1.12 to $0.86 \mathrm{~V}$ ). The lowest decrease in the overvoltage 

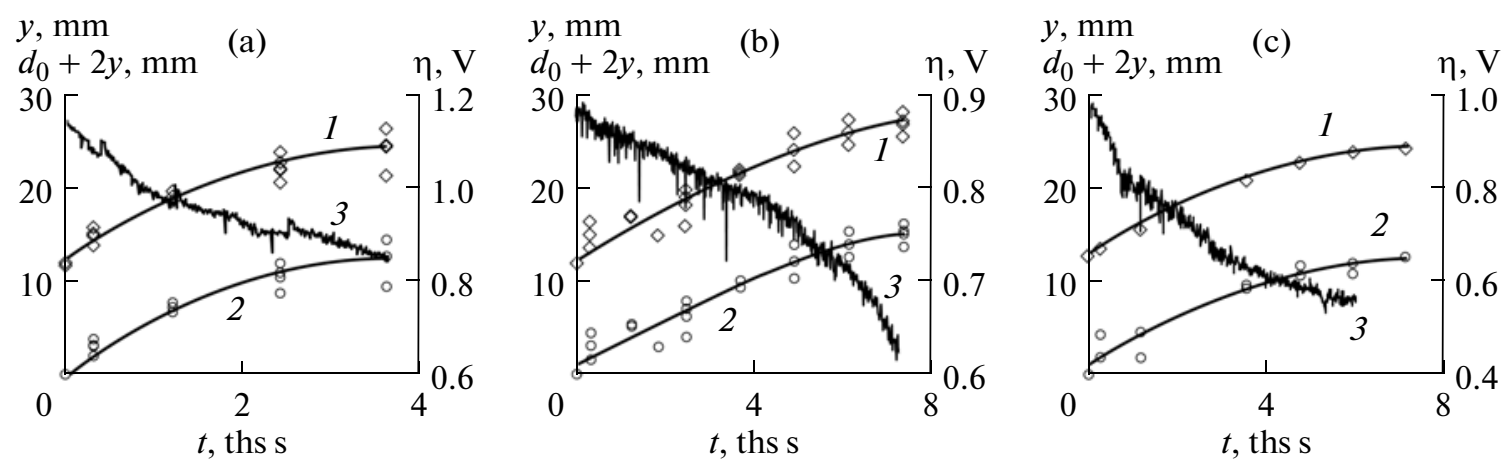

Fig. 2. Variation in time of $(1)$ the electrode diameter with the precipitate $\left(d_{0}+2 y\right),(2)$ the height of the dendritic layer, and (3) the cathode overvoltage ( $\eta$ ) for (a) PML0, (b) PMS-1, and (c) GG depositions.

before the removal from the cathode is observed for the growth of the GG deposition (down to $0.57 \mathrm{~V}$ ).

The close dynamics of the development of dendrites (Fig. 2) still does not allow us make a conclusion about the identity or the radical distinction in the structure of these precipitates and its possible change during the electrolysis. If structural characteristics $N r_{\mathrm{t}}^{2}$ are invariable during electrolysis, then, according to expression (2), straight lines in coordinates $\left(d_{0} y+y^{2}-B\right), t$ should be observed. The experimental data processing showed (Fig. 3) that the straightlinear relationships are not retained for the depositions of all brands. For the prolonged growth period of depositions, the experimental relationships remain close to linear. However, substantial distinctions are noticeable to the end of the development period of dendrites (before the removal of depositions). Moreover, the mentioned dependence for the crystallization of the PMS-1 powder has a tendency to accept an $S$-like form.

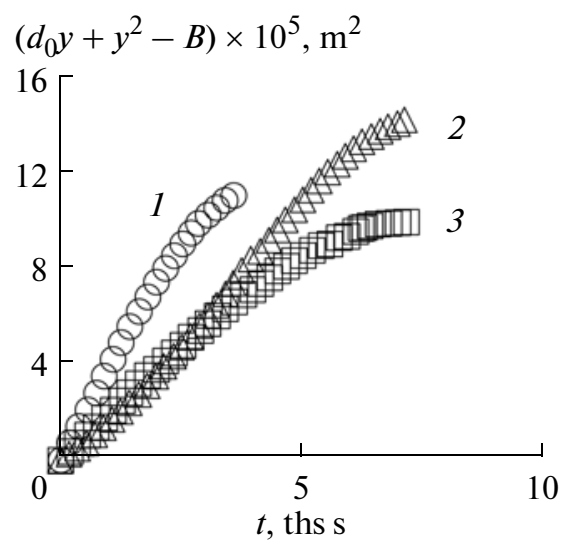

Fig. 3. Illustration of actual relationships of Eq. (2) for electrocrystallization of powders of brands (1) PML0, (2) PMS-1, and (3) GG.
The differentiation of dependences presented in Fig. 4 with respect to time makes it possible to follow the variation in the structural parameter $N r_{\mathrm{t}}^{2}$ for the powder of every brand during its electrodeposition stage. In principle, the regularities of the behavior of this structural characteristic of dendritic depositions during the electrolysis stage are similar for the powders under study, namely, its relatively small variations at the segment of the active development of dendrites are replaced by its rapid rise to the process end before the removal of depositions from the cathode. In connection with the noticeable spread of the experimental data in the lengthening dynamics of dendrites (Fig. 2), we compare the dependences $N r_{\mathrm{t}}^{2}(t)$ during the electrolysis of the powders of various brands using the variance analysis (table). We determine the variance of derivations found based on measurements of magnitudes $N r_{\mathrm{t}}^{2}$ depending on trends-approximating second-degree polynomials $\left(D_{\mathrm{t}}\right)$ with the number of degrees of freedom $f_{\mathrm{t}}$-by the number of measure-

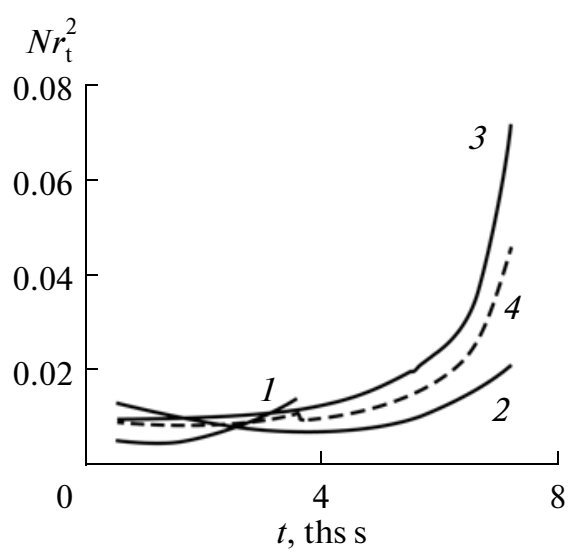

Fig. 4. Approximating lines of $N r_{\mathrm{t}}^{2}(t)$ trends for powders of brands (1) PML0, (2) PMS-1, (3) GG, and (4) the overall average line between them. 
Results of the statistic analysis

\begin{tabular}{c|c|c|c|c|c}
\hline \multicolumn{2}{c|}{ Within-group parameters } & \multicolumn{2}{c|}{ Between-group parameters } & \multicolumn{2}{c}{ Fischer criterion } \\
\hline $\begin{array}{c}\text { variance } \\
D_{\mathrm{wg}}\end{array}$ & $\begin{array}{c}\text { degree } \\
\text { of freedom } f_{\mathrm{wg}}\end{array}$ & $\begin{array}{c}\text { variance } \\
D_{\mathrm{bg}}\end{array}$ & $\begin{array}{c}\text { degree } \\
\text { of freedom } f_{\mathrm{bg}}\end{array}$ & $\begin{array}{c}\text { experimental } \\
F_{\mathrm{ex}}\end{array}$ & $\begin{array}{c}\text { tabulated } \\
F_{\mathrm{t}}(5 \%, 2,16)\end{array}$ \\
\hline $3.8 \times 10^{-5}$ & 16 & $3.19 \times 10^{6}$ & 2 & 83.83 & $3.63[8]$ \\
\hline
\end{tabular}

ments subtracting the number of coefficients of equations of the approximating polynomial. Then the approximating polynomial is found based on these relationships (Fig. 4, curve 4), which is the average between the compared ones. The ratio of the sum of quadrates of deviations for starting polynomials from the overall average gives the intergroup variance $D_{\text {inter }}$. Finally, we form the experimental Fischer criterion $\left(F_{\text {ex }}\right)$ and compare it with the tabulated $\left(F_{\mathrm{t}}\right)($ table $)$ and find that the experimental value is higher. This means that the distinctions between dependences $N r_{\mathrm{t}}^{2}(t)$ for various powder brands are more substantial than the deviations of $N r_{\mathrm{t}}^{2}(t)$ from its individual approximating polynomial. In other words, the crystallization characteristics for each powder brand are individual already at the electrolysis stage and are irreducible to the common averaged characteristics.

Due to this fact, we divide parameter $N r_{\mathrm{t}}^{2}(t)$ into $r_{\mathrm{t}}(t)$ and $N(t)$ for each powder brand separately using the kinetic characteristics of the reduction of copper corresponding to every brand ( $i_{0}$ is the exchange current density, $\alpha$ is the transfer coefficient, and $i_{\lim }$ is the limiting current density), which were preliminarily determined at the laboratory. According to the mixed control of the cathode process, we have the relationship for the vertexes of dendrites of the growth front [3]:

$$
i_{\mathrm{t}}=i_{\mathrm{kin}} i_{\text {lim.sph }} /\left(i_{\mathrm{kin}}+i_{\text {lim.sph }}\right),
$$

in which the kinetic current density for copper $i_{\text {kin }}(t)$ is calculated using parameters $i_{0}, \alpha$ and the data of chronopotentiograms (Fig. 2), which are approximated by the sixth-order polynomial. We find values of $i_{\mathrm{t}}$ by the differentiation of dependence $y(t)$ in combination with the Faraday law in a form $i_{\mathrm{t}}=V(z F)^{-1} d y / d t$, where $V$ is the molar volume of copper. Opening the expression for the limiting current density of the spherical diffusion as $i_{\text {lim.sph }}=z F D c / r_{\mathrm{t}}$ (where $D$ is the diffusivity of copper ions and $c$ is the concentration of these ions in the solution), we calculate the variation in quantity $r_{\mathrm{t}}$ during electrolysis and divide dependences $N r_{\mathrm{t}}^{2}(t)$ into $N(t)$ and $r_{\mathrm{t}}(t)$ (Fig. 5).

It is clearly seen from Fig. 5 that the dynamics of varying the structural parameters of dendrites (dependences $r_{\mathrm{t}}(t)$ and $\left.N(t)\right)$ is strictly individual for every powder brand. For example, the radius of tips for the PML0 brand increases from 0.2 to $0.4 \mu \mathrm{m}$ only after 2000 s of electrolysis; dendrites of the PMS-1 powder retain $r_{\mathrm{t}} \sim 0.3 \mu \mathrm{m}$ for $5000 \mathrm{~s}$ of electrolysis; and, in the deposition for the GG powder, $r_{\mathrm{t}}$ varies from 2 to $>10 \mu \mathrm{m}$. This means that the dendritic depositions for every powder brand have their own structural characteristics already at the electrolysis stage. The monitoring procedure used for this part of the investigation has a too low frequency of measuring the diameter of the electrode with the deposition: once for 15-20 min. The obligatory cathode elevation undoubtedly violates the development dynamics of the deposition, which leads to the spread of the $y(t)$ data. (a)

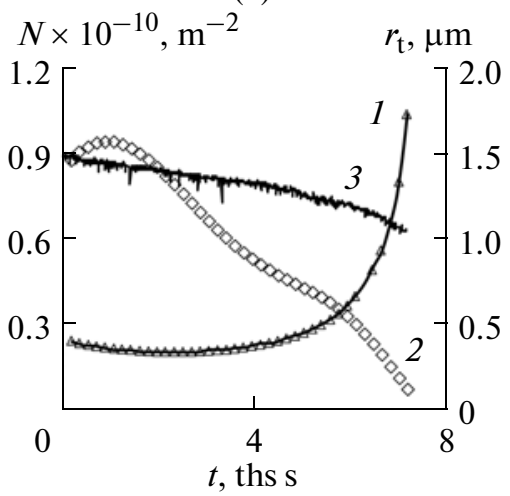

(b)

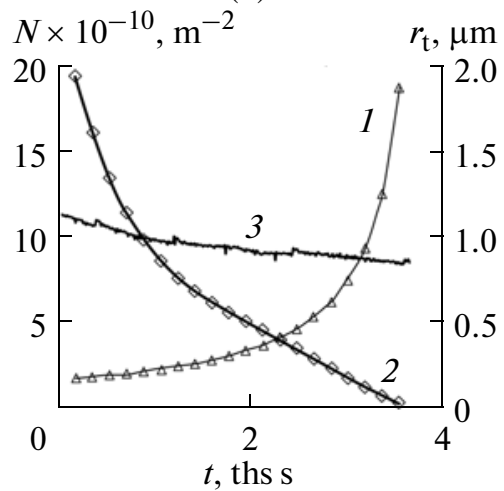

(c)

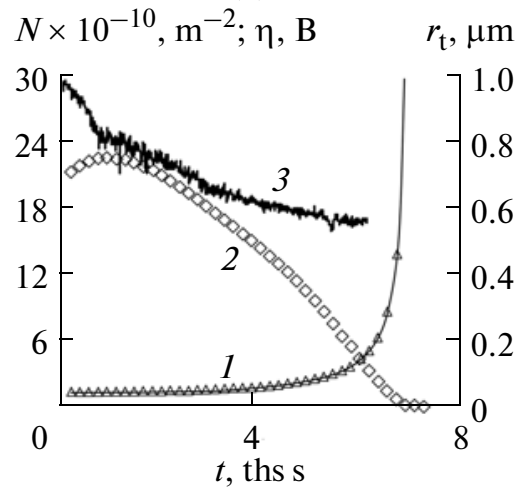

Fig. 5. Variation at the electrolysis stage of quantities (1) $r_{\mathrm{t}}$, (2) $N$, and (3) $\eta$ when producing (a) PMS-1, (b) PML0, and (c) GG powders. 
(a)

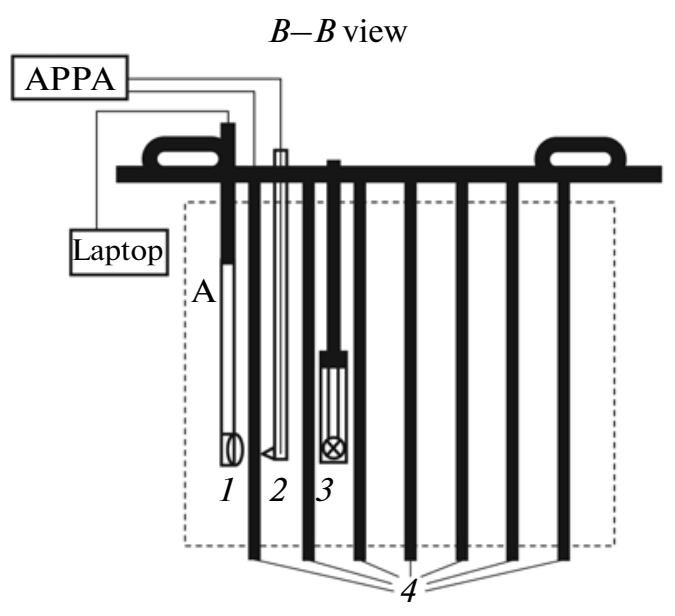

(b)

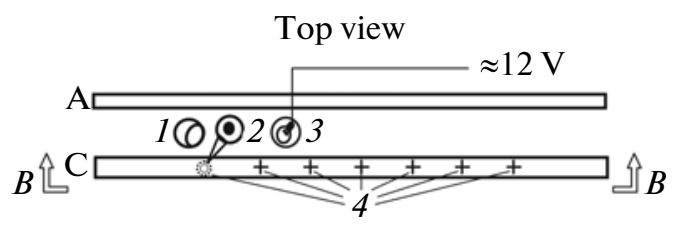

Fig. 6. (a) Layout of measuring the cathode overvoltage with (b) top view, which shows the arrangement of video recording devices during the electrolysis. A is the anode sheet and $\mathrm{C}$ is the cathode bar with rods. Explanations can be found in the text.

The following part of the investigation was devoted to developing the method (Fig. 6) of immediately video recording the deposition development by immersed video camera 1 ("e-Messenger 112") connected with a laptop (computer) and placed into a glass thermally resistant cylinder. The cable of the web chamber is placed into a vinyl tube, which is inserted into a sealing rubber ring. The upper part of the cylinder is encapsulated with silicon. Halogen lamp 3 for illumination is placed into a glass thermally resistant vessel, which is encapsulated similarly to the web camera. The camera and the lamp are lowered into the electrolyzer. A beak of electrolytic key 2 is approached to the cathode rod 4 monitoring the location by the image on the laptop screen. After tuning and preparation of the APPA multimeter, the current is switched on with the continuous recording of the overvoltage and the video image removing the shunt between the cathodic and anodic bars. Recording is stopped as the growth period of the deposition is finished. The further processing of the experimental data is performed by the above-described method (the transfer of the electric data from the APPA into the computer and the frame-by-frame decoding of the video record).

The purpose and task of this experimental study were the statistic evaluation of the reproducibility of

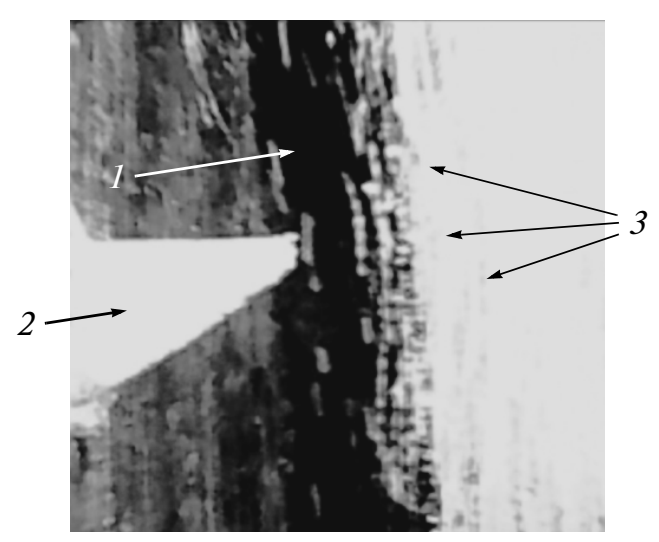

Fig. 7. Segment of the cathode rod upon switching on the current. (1) Cathode, (2) beak of the electrolytic glass key, and (3) traces of the bubbles of liberated hydrogen.

the results describing the developmental dynamics of the loose deposition (the powder of the PMS-1 brand), the calculation of the variational dynamics of structural characteristic $N r_{\mathrm{t}}^{2}(t)$ according to (2), and the measurement of $r_{\mathrm{t}}^{2}(t)$ and $N(t)$ using the kinetic parameters of reduction of copper and measured chronopotentiograms $\eta(t)$.

\section{EXPERIMENTAL RESULTS}

Intense gas liberation at the rods $12 \mathrm{~mm}$ in diameter starts immediately after the current is switched on (Fig. 7). The flow of liberated hydrogen was fixed in a form of traces and separate bubbles. In most cases, the bubble diameter is so small that the traces in the image merge into a white fog of gas elevating on the electrolyte surface (Fig. 7).

According to a series of photographs made while processing the video record of the growth dynamics of the dendritic deposition (Fig. 8), the hydrogen liberation intensity considerably lowers as the dendritic deposition grows. According to [6], a decrease in velocity $V_{\mathrm{H}_{2}}$ leads to an increase in the thickness of the diffusion layer $\delta_{\mathrm{ef}}$ :

$$
\delta_{\text {ef }}=\frac{v^{0.013} D^{0.487}}{0.93} \sqrt{\frac{d_{\mathrm{bub}}}{v_{\mathrm{H}_{2}}}},
$$

where $v$ is the kinematic viscosity of the solution and $D$ is the diffusivity.

Thus, by almost $60 \mathrm{~min}$ of electrolysis, the effect of the stirring action, which is exerted by hydrogen on the near-electrode space, is the determining factor in the formation of the structure of the dendritic deposition. To the end of its growth period, hydrogen almost stops liberating, while an increase in the cathode diameter compared with the precipitate retards. The calculation of thickness $\delta_{\text {ef }}$ according to relationship (8) showed 


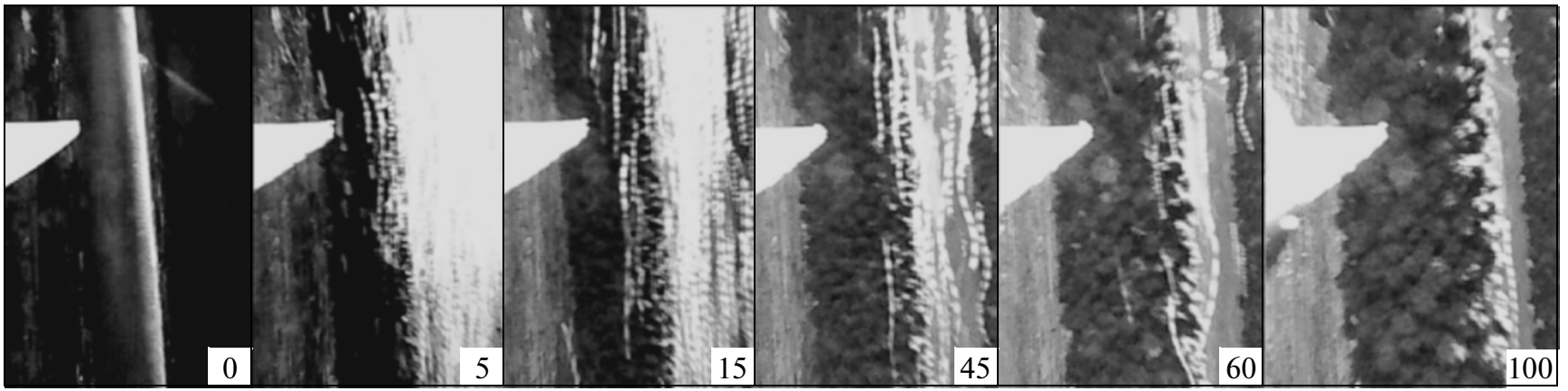

Fig. 8. Development of the dendritic layer of the PMS-1 deposition in time. The electrolysis duration from the instant of switching on the current is given (in minutes) in the lower corner of each frame.

that an increase in the bubble diameter $\left(d_{\text {bub }}\right)$ to $300 \mu \mathrm{m}$ leads to $\delta_{\text {ef }}$ close to the thickness of the stationary diffusion layer, while it is possible to measure only the individual bubbles no smaller than 300$500 \mu \mathrm{m}$ in size. At the same time, thin "fog" strips, which are formed by the flow of liberated hydrogen, remain near the electrode. In connection with this, we evaluated diameter $d_{\text {bub }}$ as the thickness of such strip constituting $0.15 \mu \mathrm{m}$. This value of $d_{\text {bub }}$ is accepted as independent of time. As the dendrites lengthen, the area of the growth front increases, which decreases hydrogen liberation velocity $V_{\mathrm{H}_{2}}$.

The sequential measurement of the electrode diameter with the growing deposition (Fig. 9) allows

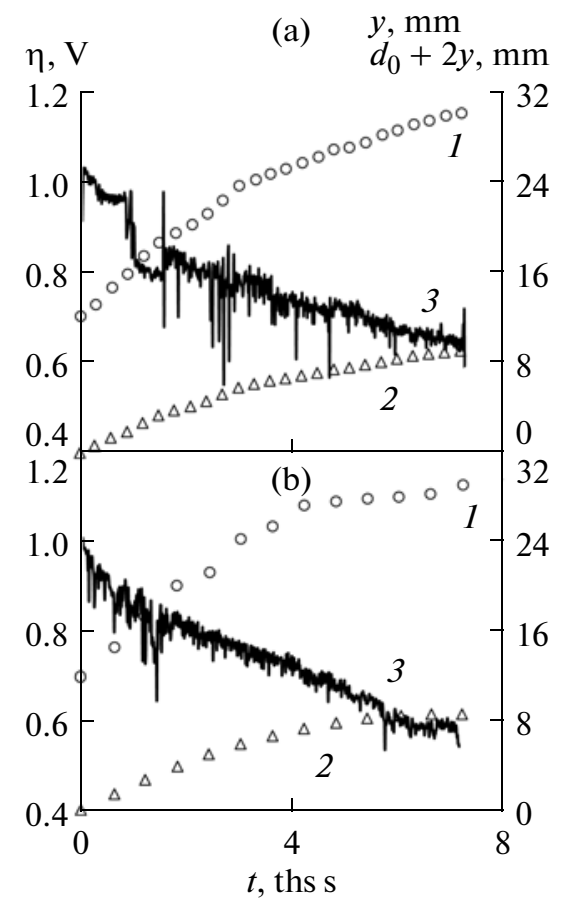

Fig. 9. Temporal dependences of (1) $d_{0}+2 y$, (2) $y$, and (3) $\eta$ as a result of two parallel experiments (a) and (b) of the growth of the PMS-1 depositions. us to find the time dependence of the dendrite length, while the multimeter readings ensure the record of chronopotentiograms $\eta=f(t)$.

\section{RESULTS AND DISCUSSION}

We evaluated the reproducibility of the results of parallel experiments using the variance analysis of the data $d_{0}+2 y=f(t)$, which describes the developmental dynamics of the precipitate in producing the powder of the PMS- 1 brand. We compared the variance of deviations of experimentally measured $d_{0}+2 y$ from the second-degree polynomials (within-group variance $D_{\mathrm{wg}}$ ) with the between-group variance $D_{\mathrm{bg}}$, which is formed as the sum of quadrates of deviations of approximating polynomials from the overall average polynomial. The sum of within-group variances of three experiments $D=$ $1.2251 \mathrm{~mm}^{2}$ with a number of degrees of freedom of 29 . The approximating second-degree polynomials are used to find dependences $d_{0}+2 y=f(t)$ averaged by three experiments (Fig. 10) and between-group dispersion $D_{\text {bg }}=3.1749 / 2=1.5874 \mathrm{~mm}^{2}$.

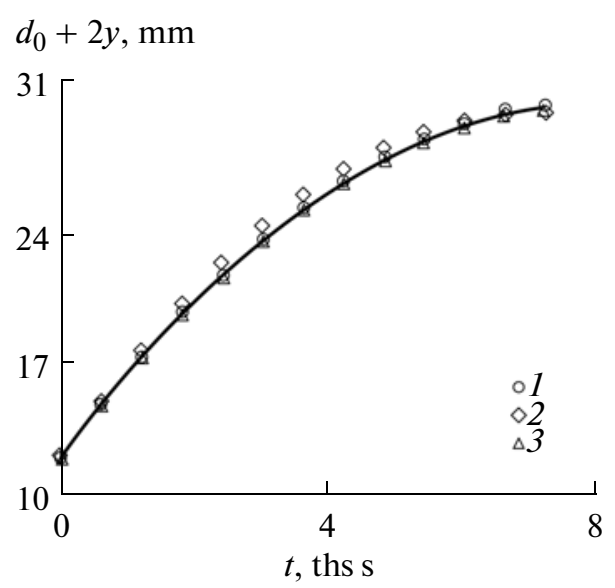

Fig. 10. Experimental trends $(1-3)$ and the overall analytical curve $d_{0}+2 y=f(t)$ (solid line) for the series of three experiments. 


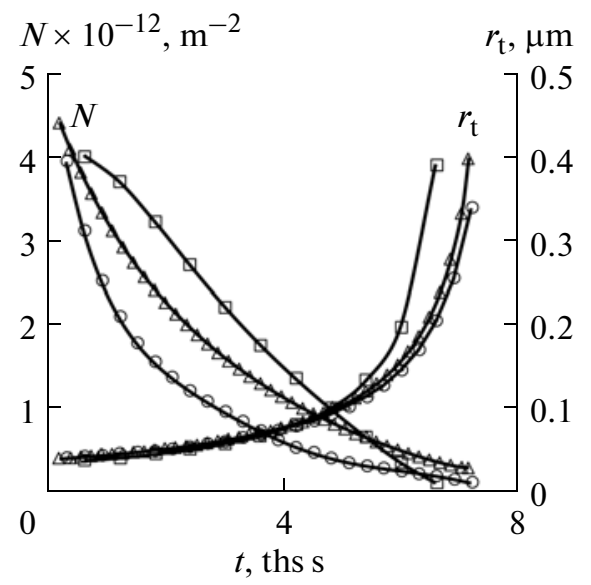

Fig. 11. Variation in time of the radius of tops and their densities in three parallel experiments.

Thus, the experimental value of the Fischer criterion was $F_{\mathrm{ex}}=1.5874 / 1.2251=1.2957$, with numbers of degrees of freedom of 2 and 29. The tabulated value of the Fischer criterion for the significance level of $5 \%$ and numbers of degrees of freedom of 2 and 29 is $F_{\mathrm{t}}=$ 3.3158. The experimental value of the criterion is lower than the tabulated one. The within-group variances are uniform, while the results of the investigation into the growth dynamics of the layer of the dendritic deposition are reproducible. According to Eq. (2), the dependence of the derivative with respect to time for expression $d_{0} y+y_{2}-B$ makes it possible to find the variation in time of the structural parameter of dendritic precipitate $N r_{\mathrm{t}}^{2}$ as well as the variations in $r_{\mathrm{t}}$ and $N$ during the electrolysis (Fig. 11). The density of growing tips on the growth front rapidly lowers to the growth end of the loose precipitate and their radius increases very rapidly. The structural elements of the dendritic depositions lose their branching (a decrease in $N$ ) and acquire a massive form of spherolites (a rapid increase in $r_{\mathrm{t}}$ ). It follows from Fig. 11 that such a phenomenon occurs somewhat earlier than the complete stopping of hydrogen evolution, which is observed with a decrease in overvoltage to $0.55 \mathrm{~V}$ [7]. In connection with such dynamics of varying the deposition structure, the period of its continuous growth should be shortened to 6000-6600 s in order to avoid the probable intergrowth of the upper layer of the dendritic deposition.

\section{CONCLUSIONS}

The structure of the dendritic depositions of various brands differs already at the electrolysis stage, which should be taken into account when composing the charge to produce wares with specified characteristics. According to the results of investigations, using the procedure of continuous monitoring of the electrolysis with the use of video recording immediately in industrial conditions, it is recommended to shorten the duration of the continuous crystallization of the deposition during the electrolysis by 15-20 min.

\section{ACKNOWLEDGMENTS}

This study was supported by the Russian Foundation for Basic Research, project no. 11-03-00226.

\section{REFERENCES}

1. Handbook on Non-Ferrous Metal Powders, Neykov, O.D., Naboychenko, S.S., and Dowson, G., LondonAmsterdam-NewYork: Elsevier, 2009.

2. Lebed', A.B., Yakovleva, L.M., Sultangareeva, E.E., et al., in Aktual'nye problemy elektrokhimicheskoi tekhnologii. Sbornik statei molodykh uchenykh (Topical Problems of Electrochemical Technology. Collected Articles of Young Scientists), Saratov: Saratov State Univ., 2005, p. 144.

3. Murashova, I.B., Darintseva, A.B., and Rudoi, V.M., Elektrokhimiya, 2010, vol. 46, no. 6, p. 1.

4. Murashova, I.B., Sokolovskaya, E.E., and Lebed', A.B, Tsvetn. Met., 2007, no. 10, p. 46.

5. Murashova, I.B., Taushkanov, P.V., and Burkhanova, N.G., Elektrokhimiya, 1999, vol. 35, no. 7, p. 46.

6. Yakubova, T.V. and Murashova, I.B., Elektrokhimiya, 1995, vol. 31, no. 3, p. 483.

7. Sokolovskaya, E.E., Murashova, I.B., Lebed', A.B., et al., Tsvetn. Met., 2010, no. 3, p. 33.

8. Bol'shev, L.N. and Smirnov, N.V., Tablitsy matematicheskoi statistiki (Mathematical Statistics Tables), Moscow: Nauka, 1983.

Translated by N. Korovin 